\title{
Any Metastatic Diagnosis Absent
}

National Cancer Institute

\section{Source}

National Cancer Institute. Any Metastatic Diagnosis Absent. NCI Thesaurus. Code C159615.

An indication that metastatic disease has not been diagnosed. 